C-A/AP/\#211

June 2005

\title{
Maps for Coupled Electron and Ion Clouds in Accelerators
}

\author{
Ubaldo Iriso and Stephen Peggs
}

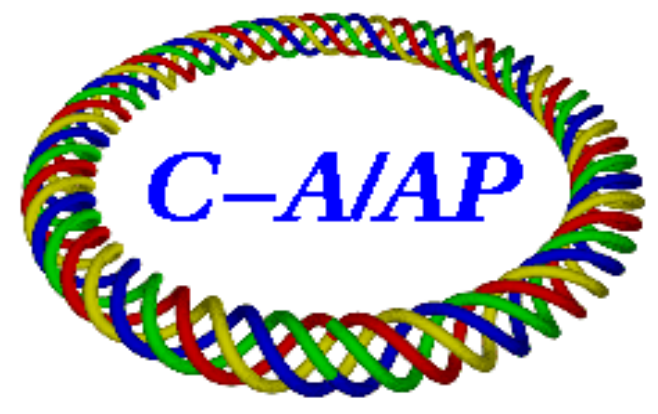

Collider-Accelerator Department Brookhaven National Laboratory Upton, NY 11973 


\title{
Maps for Coupled Electron and Ion Clouds in Accelerators
}

\author{
Ubaldo Iriso* and Stephen Peggs ${ }^{\dagger}$ \\ Brookhaven National Laboratory, Upton, NY
}

(Dated: March 2, 2005)

\begin{abstract}
Electron clouds sometimes limit accelerator performance, by their appearance when the circulating bunch population exceeds a threshold value. Dynamical models of cloud build-up, and of the phase transition from "cloud off" to "cloud on", are enhanced when simple coupling between electron and ion clouds is included. Maps are then capable of reproducing the first order phase transitions seen in practice. They also predict that hysteresis, period doubling, and chaotic phenomena may be observed.
\end{abstract}

PACS numbers: 29.20.Dh

Quasi-stationary seed electrons in the vacuum pipe of an accelerator are accelerated in an impulse to an energy of order $1 \mathrm{keV}$ when a bunch of positive particles passes by. These electrons strike the vacuum pipe wall, rapidly dissipating and diffusing into an electron spectrum that has a typical energy of only a few eV, until the next bunch passes by, when the process is re-iterated. Under some conditions electron multiplication occurs, and an electron cloud rapidly builds, until a stable dynamic equilibrium is attained. The necessary conditions include vacuum chamber surface characteristics (mainly given by the dependence of the secondary emission yield on the incident electron energy), beam pipe geometry, and beam characteristics. For a given bunch spacing and for a particular beam pipe, electron clouds only build if the bunches have a large enough positive charge.

Electron clouds have been observed in many accelerators, often acting as a fundamental limit to machine performance through dynamical instabilities, unacceptably large associated vacuum pressure increases, or cryogenic heat loads [1-4]. Experimental data shown in Fig. 1 illustrate how both first and second order phase transitions are seen in the Relativistic Heavy Ion Collider (RHIC), as a threshold bunch population is crossed.

Electron cloud evolution is modeled with some significant success using complex simulation codes, typically tracking individual electrons or macro-particles, and sometimes employing 3-dimensional finite-element methods to calculate self-consistent forces and fields [6]. Under some conditions it is found that the simulated evolution from the passage of bunch $m$ to $m+1$ is empirically well represented by a cubic map

$$
\rho_{m+1}=(1+a) \rho_{m}+b \rho_{m}^{2}+c \rho_{m}^{3}
$$

where $\rho[\mathrm{nC} / \mathrm{m}]$ is the linear electron cloud density $[4,7]$. Weak electron clouds grow exponentially in time if $a>0$

$$
\rho_{m} \approx \rho_{0} e^{a m}
$$

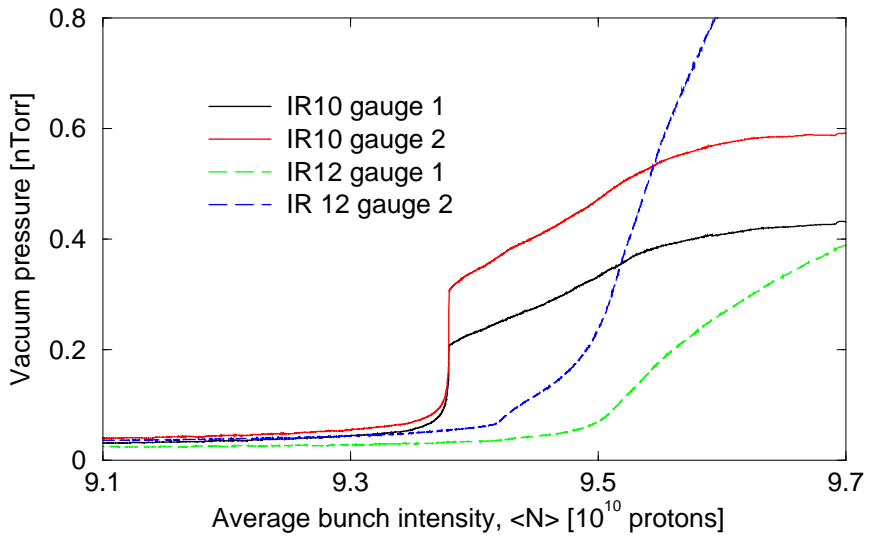

FIG. 1: First and second order electron cloud phase transitions observed in the 10 o'clock and 12 o'clock interaction region straights of the Relativistic Heavy Ion Collider. The data were taken as the bunch population slowly decayed during beam fill 5905. The pairs of gauges in IR10 and IR12 are about $15 \mathrm{~m}$ and $1 \mathrm{~m}$ apart, respectively. Enhanced vacuum pressures are correlated with the direct observation of electron clouds [5]. The actual copper ion bunch population distribution is converted to an equivalent average number of protons per bunch.

This growth is often fast $-a$ is of order 1 - but it is limited, so that a stable equilibrium is obtained when

$$
\rho_{m+1}=\rho_{m} \equiv \rho_{s}
$$

For example, if the cubic term in $c$ is negligible, then

$$
\rho_{s}=a /(-b)
$$

A negative value of $b$ is physically sensible, representing the self-limiting influence of accumulated (negative electron) space charge.

The coefficient $a$ increases monotonically with the bunch population $N$, so that the stable electron cloud density $\rho_{s}(N)$ is also a function of bunch population. Equation 4 then predicts that the phase transition from electron cloud "off" to "on" is second order $-\rho_{s}(N)$ increases smoothly from zero above a critical threshold population, when $a$ becomes positive. Complex simulation codes consistently reproduce only second order phase
*Electronic address: ubaldo@bnl.gov

${ }^{\dagger}$ Electronic address: peggs@bnl.gov 
transitions [8]. The failure to reproduce the first order phase transitions seen in RHIC (see Fig.1) indicates that there is missing physics in the simulations and the modeling.

A likely candidate for additional physics, the interplay between electron clouds and positive ion clouds, was first introduced to explain experimental observations at RHIC [9], and was suggested more recently as a future concern for the Large Hadron Collider [10]. Models of this interplay face two main challenges: a significant number of uncertain surface physics parameters for both electron and ions, and extremely different time scales for electron and ion cloud dynamics. Long ion lifetimes imply very long CPU times for simulations. Not only is the typical time-of-flight between surfaces much longer for a massive ion than for an electron of similar kinetic energy, but also the backscattering probability for ion energies below about $30 \mathrm{eV}$ is close to unity [11]. The lifetime of such ions is not characterized by their time-of-flight, as with electrons, but by vacuum pumping times, usually measured in seconds. Therefore, electron clouds evolve with a lifetime on the order of 1 microsecond, while ion clouds evolve between three and six orders of magnitude more slowly.

It is relatively easy to add the interplay between electron clouds and ion clouds to a map model. For example, consider the "proof-of-principle" coupled maps

$$
\begin{aligned}
\rho_{m+1} & =\left(1+a+b \rho_{m}+y R_{m}\right) \rho_{m}+c \rho_{m}^{3} \\
R_{m+1} & =(1+A) R_{m}+Y \rho_{m}
\end{aligned}
$$

where $R_{m}[\mathrm{nC} / \mathrm{m}]$ is the ion cloud density after the passage of the $m$ 'th bunch. (Both $\rho$ and $R$ are defined to be positive.) If the coupling coefficients are turned off $(y=Y=0)$, then the electron cloud map Eqn. 1 is recovered, along with the uncoupled ion map

$$
R_{m+1}=(1+A) R_{m}
$$

The negative coefficient $A$ represents the rate that the ion cloud clears, for example through vacuum pumping and neutralization. Since the massive ion clouds only clear slowly, it is expected that $-A \ll 1$. There are two coupling mechanisms in Eqns. 5 and 6:

1. First, electrons collide with the rest gas in the vacuum chamber to create a positive ion cloud. This is represented by the term $Y \rho_{m} . Y$ is positive, but its order of magnitude is not trivially apparent.

2. Second, the slow moving positive ions tend to neutralize the negative electron space charge of the accumulated electron cloud. This is represented by the term $y R_{m}$. Physical values of $y$ are positive, and of the same magnitude as $b$, so $y \sim|b|$.

Stationary equilibrium occurs (if it does) when

$$
\begin{aligned}
\rho_{m+1} & =\rho_{m} \equiv \rho_{s} \\
R_{m+1} & =R_{m} \equiv R_{s}
\end{aligned}
$$

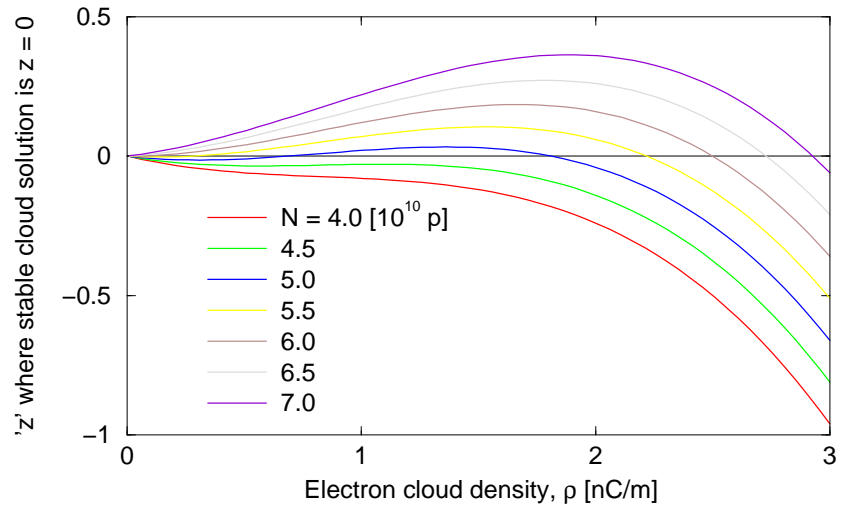

FIG. 2: Graphical solution of the saturated electron cloud density for a family of different bunch intensities, $N$, when $(b, c, y, A, Y)=(-0.1,-0.08,0.4,-0.04,0.03)$.

at electron cloud densities that are the roots of the cubic equation

$$
0=a \rho_{s}+\tilde{b} \rho_{s}^{2}+c \rho_{s}^{3}
$$

where the "effective space charge coefficient"

$$
\tilde{b}=b-(y Y / A)
$$

has been introduced. One of the $3 \operatorname{roots}\left(\rho_{s}=0\right)$ is trivial. The 2 nd and 3 rd roots,

$$
\rho_{s}=\frac{-\tilde{b} \pm \sqrt{\tilde{b}^{2}-4 a c}}{2 c}
$$

are only physical if their values are real and positive. If so, one stationary solution is dynamically stable, while the other is unstable. The stable ion density is simply related to the stable electron density:

$$
R_{s}=-(Y / A) \rho_{s}
$$

(recall that $Y$ is positive and $A$ is negative).

Figure 2 graphically illustrates the case when all of the coupled map coefficients are constants except for the weak electron cloud growth rate $a$, which is presumed to depend linearly on the bunch population according to

$$
a=-0.6+0.1\left(N / 10^{10}\right)
$$

(The coupled map coefficients quoted in the caption, and used in all subsequent Figures, are illustrative - they are not intended to quantitatively reproduce RHIC results.) The crucial effect of the coupling is to change the sign of the effective space charge coefficient $\tilde{b}$ - positive ions neutralize the negative space charge of the negative electrons at intermediate bunch populations, permitting two physical stationary solutions. For example, a stable solution exists at $\rho_{s} \approx 1.8[\mathrm{nC} / \mathrm{m}]$, as well as an unstable solution at $\rho_{s} \approx 0.7[\mathrm{nC} / \mathrm{m}]$, when $N=5.0 \times 10^{10}$. No (non-zero) stationary solution is possible at a slightly smaller bunch population of $N=4.5 \times 10^{10}$. 


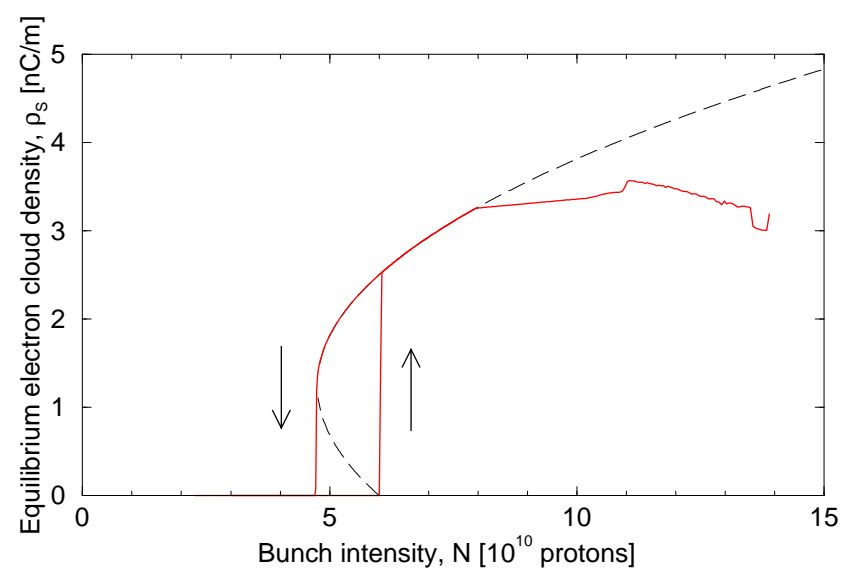

FIG. 3: Evolution of the electron cloud density as the bunch population $N$ is first slowly decreased, and then slowly increased. The precipitous and hysteretic behavior is typical of first order phase transitions. The dashed lines represent the two stationary solutions described in Eqn. 12.

These conditions lead to a first order phase transition, and to hysteresis. Fig. 3 shows the results of a dynamical simulation, in which the coupled maps are applied directly, first as the bunch population is slowly decreased, and then as it is slowly increased. The solid line shows that the stable electron cloud density decreases as the bunch population is reduced, until at $N \approx 4.7 \times 10^{10}$ the electron cloud collapses catastrophically. When the bunch population is then slowly increased, no electron (or ion) cloud forms up to a population of $N=6.0 \times 10^{10}$, when $a$ becomes positive and the cloud grows rapidly to the stable stationary value.

The coupled maps can be rewritten

$$
\begin{aligned}
\Delta \rho / \Delta t & =(a+b \rho+y R) \rho+c \rho^{3} \\
\Delta R / \Delta t & =A R+Y \rho
\end{aligned}
$$

where the nominal time step $\Delta t=1$ corresponds to the passage of a single bunch. The coupled differential equations that are obtained in the limit that $\Delta t \rightarrow 0$ exhibit stable cloud solutions that do not depart from the classical stationary solutions of Eqn. 12. By contrast, Fig. 3, with $\Delta t=1$, shows agreement with the classical stationary stable solution (upper dashed line) only up to about $N=8.0 \times 10^{10}$, above which different dynamical phases become active.

Figure 4 shows the evolution of the electron and ion clouds for different bunch populations, always starting with the same (arbitrary) initial cloud densities. The clouds decay away or build to stable solutions with $N=$ $3 \times 10^{10}$ and $6 \times 10^{10}$, consistent with classical expectations, and as shown in Fig. 3. However, the clouds evolve into a stable period-2 oscillation when $N=9 \times 10^{10}$ protons per bunch. Figure 5 takes a closer look at the chaotic dynamics that evolve when $N=12 \times 10^{10}$.

Difference equations are inherently richer than the

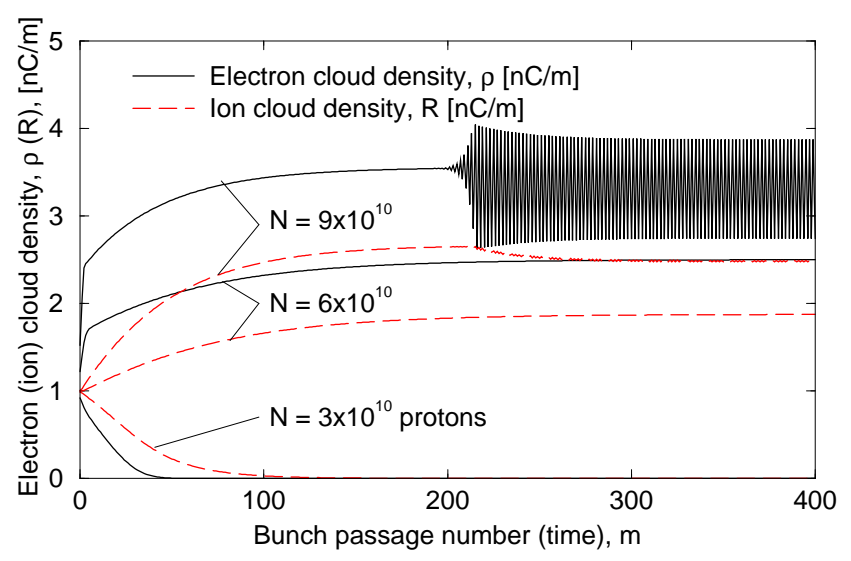

FIG. 4: Dynamical evolution of the electron and ion cloud densities as a function of time (bunch passage number) for 3 different bunch intensities, $N=1 \times 10^{10}, 2 \times 10^{10}$, and $3 \times 10^{10}$ protons per bunch.

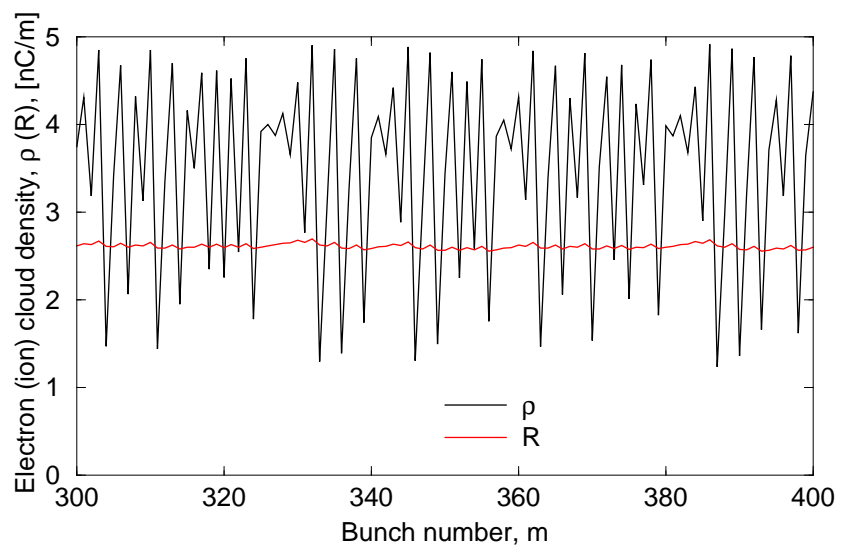

FIG. 5: Chaotic behavior of electron and ion cloud densities when $N=12 \times 10^{10}$ protons per bunch.

analogous differential equations, in the dynamical behavior that they display. Coupled maps can generate period doubling and chaos, behavior that does not occur in the smoothed world of differential equations. Such additional dynamical phases have not (yet) been observed in electron clouds in accelerators, but it is possible they occur at, or near, typical operating conditions. An understanding of coupled cloud dynamics from the map perspective may prove important in enhancing accelerator performance.

Bunch-by-bunch maps are more appropriate than differential equations in modeling coupled cloud dynamics, because of the rapid evolution of the electron cloud - the high frequency components - after the violent transient of a bunch passage. In particular, the electron cloud energy spectrum changes enormously between bunch passages, with typical electron energies dropping from of order $1 \mathrm{keV}$ to a only a few eV.

The "proof-of-principle" coupled maps presented here 
can generate electron and ion clouds that turn "on" and "off" precipitously. Such first order phase transitions are sometimes seen in practice, but are beyond the capability of contemporary simulation codes, which model elec- tron clouds in isolation. Other coupling mechanisms than those presented here are also plausible, and can be modeled in the map formalism.
[1] Electron cloud measurements and simulations for the Brookhaven Relativistic Heavy Ion Collider, W. Fischer, M. Blaskiewicz, M. Brennan, and T. Satogata, PRST-AB 5, 124401 (2002).

[2] CERN SPS electron cloud heat load measurements and simulations V. Baglin and B. Jenninger. PRST-AB 6, 063201 (2003).

[3] Review of single bunch instabilities driven by an electron cloud, F. Zimmermann, PRST-AB 7, 124801 (2004).

[4] RHIC Electron Clouds, U. Iriso, PhD Thesis, to be published, U. of Barcelona, (2005).

[5] Electron induced molecular desorption during multipacting in the RHIC beam pipes, U. Iriso and W. Fischer, C-AD/AP/191, Upton, February 2005.

[6] Review and Comparison of Simulation Codes Modeling Electron-Cloud Build Up and Instabilities, E. Benedetto et al, Proc. of EPAC'04, Lucerne, 2004. See also http://wwwslap.cern.ch/collective/ecloud02/ecsim

[7] Maps for electron clouds U. Iriso and S. Peggs, PRST-AB 8, 024403 (2005).

[8] Electron cloud phase transitions, U. Iriso and S. Peggs,
C-AD/AP/147, Upton, April 2004.

[9] Electron Cloud driven vacuum instability, W. Fischer, U. Iriso, and E. Mustafin. Proc. of ICFA Workshop on High Intensity and High Brightness Hadron Beams, Bensheim, October 2004.

[10] Vacuum aspects for an LHC upgrade, O. Gröbner, Proc. of CARE HHH-2004 Workshop, Geneva, November 2004.

[11] The physical basis of ultrahigh vacuum. P.A. Redhead, J.P. Hobson, and E.V. Kornelsen. High vacuum series, Ed. by L. Holand, Ch 4, pp 187, 1968.

\section{Acknowledgments}

We wish to acknowledge the support of many people in the Collider-Accelerator Department at Brookhaven National Laboratory, including M. Blaskiewicz, W. Fischer, R. Hseuh, and S.Y. Zhang. 